\title{
Caracterización de dos experiencias exitosas de negocios inclusivos en Colombia ${ }^{1}$
}

\author{
Characterization of two successful experiences in inclusive \\ business in Colombia
}

Recibido: 08 de diciembre de 2014 - Revisado: 19 de enero de 2015 - Aceptado: 08 de abril de 2015

\author{
Edimer Leonardo Latorre Iglesias ${ }^{2}$ \\ Cindy Caterine Díaz Areiza ${ }^{3}$ \\ Angela Maria Plata Rangel ${ }^{4}$ \\ Susana Paola Marín ${ }^{5}$
}

\section{Resumen}

El reporte de caso que se presenta a continuación revisa dos experiencias de negocios inclusivos exitosas, las cuales permiten evidenciar las nuevas tendencias de responsabilidad social empresarial y sus formas de hibridarse con otros campos científicos, tales como la economía del bien común y la resiliencia empresarial. La metodología implicó el uso de herramientas cualitativas y cuantitativas con base en la etnografía virtual, para entender las particularidades de dos firmas que marcan la diferencia en su forma de hacer negocios.

\section{Palabras clave}

Negocios inclusivos, responsabilidad social empresarial, economía del bien común, resiliencia empresarial, negocios en la base de la pirámide.

\section{Abstract}

The case report presented here reviews two experiences of successful inclusive business, which allow to highlight new trends in corporate social responsibility and its forms of hybridizing with other scientific fields such as the common good economy and corporate resilience. The methodology involved using qualitative and quantitative tools based on virtual ethnography to understand the peculiarities of two firms that make a difference in the way of doing business.

\section{Keywords}

Inclusive business, corporate social responsibility, common good economy, corporate resilience, business at the base of the pyramid.
${ }^{1} \mathrm{El}$ artículo pertenece al proyecto "Investigaciones sociojurídicas" avalado y patrocinado por la Escuela de Derecho de la Universidad Sergio Arboleda Seccional Santa Marta.

${ }^{2}$ Doctor en Sociología Jurídica e Instituciones Políticas. Docente investigador de la Escuela de Derecho de la Universidad Sergio Arboleda. Actualmente es director del Grupo de Investigación Joaquín Aarón Manjarrés categorizado en la reciente medición de Colciencias en A, Universidad Sergio Arboleda, Santa Marta, Colombia. Correo electrónico:

edimer.latorre@usa.edu.co

${ }^{3}$ Abogada especialista en Derecho Penal y Criminología, especialista en Derecho Constitucional. En la actualidad es candidata a magíster en Teoría del Derecho de la Universidad Externado de Colombia y directora del Centro de Investigación Sociojurídica de la Corporación Universitaria del Caribe (Cecar), Sincelejo, Colombia. Correo electrónico:

cindy.diaza@cecar.edu.co

${ }^{4}$ Ph.D. (C). en proyectos en medio ambiente. M.Sc. en Environmental sciences. Subdirectora del Instituto de Estudios y Servicios Ambientales (IDEASA), Universidad Sergio Arboleda, Bogotá, Colombia.

Correo electrónico: angela.plata@usa.edu.co

${ }^{5}$ MBA. Universidad del Norte. Directora oficina El Rodadero del Banco Davivienda, Santa Marta, Colombia. Correo electrónico: susanapaolamarin@hotmail.com Para citar este artículo: Latorre, E., Díaz, C., Plata, A., \& Marín, S. (2015). Caracterización de dos experiencias exitosas de negocios inclusivos en Colombia. Revista $\mathrm{Ci}$ vilizar Ciencias Sociales y Humanas, 15(28), 197-212. 


\section{Introducción}

El reporte de caso que se presenta a continuación, recoge con la caracterización de dos experiencias exitosas, las diferentes acciones que promueven dos empresas colombianas, desde el enfoque de negocios inclusivos. Se persigue con esto evidenciar la posible relación entre negocios inclusivos y responsabilidad social empresarial (RSE), además de señalar las ventajas de esta modalidad, se exponen las diferentes estrategias de negocios inclusivos que estas manifiestan, como un accionar propio del nuevo paradigma de RSE.

Se aclara que los dos casos que se seleccionaron para ser estudiados, presentan un conjunto de particularidades que permitió filtrar su análisis. Estas, se pensaron desde lo exitoso de la propuesta de negocios inclusivos, que debía mostrar:

a. Sostenibilidad en el tiempo. Los casos que se escogieron muestran una curva de ascenso desde el momento de su implementación. Esta curva evidencia cualitativa y cuantitativamente un alto impacto del negocio inclusivo.

b. Impacto social económico. Los casos en análisis implican una alta huella económica en la vida de los grupos y comunidades a los cuales se direccionaron, lo que valida la capacidad de transformar realidades socioeconómicas.

c. Innovación de valor agregado. Los casos elegidos son innovadores en el entramado de casos de negocios inclusivos en Colombia. Cuentan con una o una serie de innovaciones que los hace ser altamente viables.

d. Visibilidad del negocio. El aspecto final es el reconocimiento de la opinión pública de estos dos negocios inclusivos: premios y visibilización de sus servicios.

Lo anterior exigió que el trabajo se delimitara a los actores del proceso -personas que de una forma $u$ otra son agenciadores de negocios inclusivos-. El proceso cualitativo se materializó con el uso de las siguientes herramientas: entrevistas semiestructuradas, grupos focales, observación participante, análisis de documentos y etnografía virtual, que es el seguimiento a los casos empresariales a través de sus publicaciones en la web. Lo cuantitativo se concretó en el manejo de datos y cifras que muestran los logros financieros de la organización y el impacto social de sus propuestas mediante indicadores económicos.

Es de resaltar que la etnografía virtual se construye en torno al seguimiento de las publicaciones realizadas por las dos entidades objeto de estudio, teniendo en cuenta los siguientes elementos que permitieron consolidar la información secundaria para el diseño de los casos exitosos:

- Diseño del sitio web: interactividad y accesibilidad.

- Forma de presentación de la responsabilidad social organizacional.

- Actores e instituciones involucrados en el proceso.

- Plataforma virtual y redes sociales.

- Estrategia de comunicación: construcción de marca e innovación en el diseño.

- Alineación estratégica y publicaciones.

Son estos los elementos que desde lo virtual ayudan a validar el éxito del caso, en este sentido, se exponen a continuación dos casos, los cuales se analizan en el marco de tres dimensiones: concepto relevante a explorar, contexto social en el que surge el caso y presentación de la institución e indicadores que la convierten en un caso exitoso.

\section{Un banco que no está en el lugar equivocado: servicios que apuntan a la economía del bien común}

Frente a los esquemas tradicionales del mundo capitalista y la pérdida de legitimidad de un capitalismo depredador, existen voces que 
marcan distancia y que creen profundamente en la renovación de los valores dentro del capitalismo (Felber, 2012; Porter \& Kramer, 2011; Senn, 2008; Soros, 2008; Stiglitz, 2012). En particular toma fuerza el concepto de economía del bien común. Según Christian Felber (2012), pueden impulsarse otros valores dentro de la economía. A la competencia, rendimiento, crecimiento y beneficio, podemos oponerles en la práctica comercial los valores solidarios, tales como la ayuda mutua y la cooperación.

Este profesor de economía alternativa, ofrece frente a la disyuntiva tradicional capitalismo o comunismo, un modelo de economía que favorezca a los integrantes del mismo. Una de las consignas banderas es que no podemos seguir siendo prisioneros del medio fundamental del capitalismo: el dinero. Una prueba de esto, es la abismal diferencia entre los índices de los sueldos de los gerentes y los salarios mínimos de tres países, que muestra los altos niveles de inequidad: en Austria un gerente gana 600 veces más que una persona que solo devenga el salario mínimo; en Alemania cinco mil veces más y en Estados Unidos en la industria e ingeniería financiera 65.000 y 360.000 veces más respectivamente (Felber, 2012).

En este contexto, la inequidad de renta entre ejecutivos mejor pagados y operarios pagados con el salario mínimo en las empresas pasó de 24 a 1 en el año 1965, a una diferencia de 325 a 1 en el año de 2001. En el lapso de 36 años se dio un incremento del $321 \%$ (Felber, 2012). Es por ello que, en un escenario de exclusión social, recesión económica y pobreza extrema, toma fuerza la idea de una economía del bien común. La matriz del bien común que propone Felber (2012), gira en torno a las siguientes mediciones cualitativas y cuantitativas:

1. Dignidad humana: entendida como la calidad del puesto de trabajo de una persona.

2. Solidaridad: cómo se amplía el mercado cooperativo entre los stakeholders.
3. Sostenibilidad ecológica: cómo se da la promoción del comportamiento ecológico entre los trabajadores y la comunidad.

4. Justicia social: índice de recorte de la renta y la promoción de la participación democrática y la transparencia.

En la actualidad existen 3.800 personas que se han sumado a la causa, 159 asociaciones siguen las ideas de Felber y más de 1.277 empresas usan los indicadores de la economía del bien común (Felber, 2012). En Colombia hay una compañía que propone un modelo inclusivo de negocios desde una óptica bancaria diferente y que a su manera, está desarrollando una dinámica muy similar a la propuesta de la economía del bien común, con sus indicadores de resultados demuestra que, a pesar de lo planteado para el caso de los bancos, hay entidades bancarias que pueden marcar la pauta.

El banco Davivienda forma parte del Grupo Bolívar, por tradición y al revisar con detenimiento su propuesta de cultura organizacional, llama la atención su pirámide axiológica organizacional, la cual se asienta en la creencia de que el ser humano es una fuente inagotable de valor.

En esta visión los seres humanos crean y agregan valor con sus acciones, siendo la integridad el centro de su estrategia de negocio y de su comunicación organizacional. En este panorama virtuoso de estrategia organizacional, las fortalezas, el conocimiento y el talento de más de once mil empleados se convierten en una fuerza reiterada para generar valor de manera sostenible enfocándose en sus diferentes grupos de interés.

Esta idea de lo económico, es el soporte que posibilita desplegar conceptos innovadores que revolucionan el mercado a pesar de las limitaciones de las comunidades donde se afianza su negocio. Si se hace un análisis detenido a la propuesta organizacional y a la concepción en Davi- 
vienda de lo empresarial, se podrían mencionar tres prácticas de gestión que agregan valor a los clientes y que abren nuevos mercados inclusivos de un modo socialmente responsable.

1. Planificación a largo plazo. Es una práctica de gestión que propende por responder dónde estará la organización en cinco, diez, quince o veinte años y cuáles podrían ser las consecuencias de las acciones de la compañía. De ahí, que se piense en la responsabilidad de la organización con el entorno, con los trabajadores, con los proveedores y en particular con el medioambiente. Esta visión de sostenibilidad a largo plazo implica una concepción de equilibrio entre sus stakeholders.

2. Cultura de la innovación. Su programa de desarrollo de innovaciones hasta la fecha, ha propiciado que el talento humano (más de siete mil trabajadores) haya generado por lo menos una innovación de valor agregado tecnológico; esto es gracias a que los empleados se han capacitado en gestión de la innovación (entrenadores de innovación y círculos concéntricos de innovación), permitiendo pasar de una "burocracia de gestión" a una "democracia de las ideas". El talento humano se convierte así en una fecunda fuente para enfrentar las incertidumbres del mercado actual. En Davivienda, existe de forma proactiva el "Comité de iniciativa", que consiste en una reunión mensual donde cualquier empleado que tenga una idea puede presentarla, explicar su impacto (en especial cómo impactaría positivamente el trabajo, la familia, la comunidad, el planeta o cómo podría aportar significado desde el propósito personal) y formar parte del equipo que va a llevar a cabo la tarea (Davivienda, 2013).
3. Gestión del conocimiento. Acorde con las nuevas tendencias de la gestión del conocimiento, en las bases organizacionales de Davivienda se cree en el ser humano como una fuente interminable de saber. Esto se ha logrado gracias a la creación de "equipos comando", que se encargan de aprender sobre nuevos negocios. Esta clase de coliderazgo, gira alrededor de un equipo integrado por personas de distintos departamentos con diversas habilidades e ideas que vienen de cualquier tipo de posición en la organización, para analizar nuevas oportunidades de negocio, nuevas ideas o nuevos proyectos y generar un aprendizaje sobre los mismos. Los "equipo comando" tienen autonomía y empoderamiento para estudiar, diseñar e implementar un plan de acción. En el marco de las operaciones de estos equipos es que surge DaviPlata.

DaviPlata como servicio, es un negocio inclusivo en las dimensiones teórico-prácticas que comprende el concepto. Los siete reconocimientos mundiales recibidos hasta el momento, indican que la práctica cumple con los estándares internacionales para ser concebida como negocio altamente incluyente:

- Premio de Harvard Business Review 2012 por la gestión de la innovación con impacto social.

- Premio CIT Golden Card 2012 a la innovación en medios de pago (México).

- Participante en la Cumbre Río+20 de la Organización de las Naciones Unidas del año 2012 en Brasil.

- Participante en Federación Latinoamericana de Bancos (Felaban) XI-CLAB 2011 Cartagena.

- Publicación caso de sostenibilidad DaviPlata Consejo Empresarial Colombiano para el Desarrollo Sostenible (Cecodes) 2012. 
- Reconocimiento como caso de éxito mundial de inclusión financiera por The Better Than Cash Alliance.

- Mención del caso DaviPlata por Philip Kotler, David Hessekiel y Nancy Lee (2013) en su libro Lo bueno funciona: "explica cómo las iniciativas de marketing social construyen un mundo mejor y favorecen la cuenta de resultados".

¿Que hace que DaviPlata sea inclusivo? Que ofrece una solución simple a un problema grande en Colombia: la bancarización. De acuerdo con las cifras de inclusión financiera de Henao, Borja, Mantilla, Garcia y Ordoñez (2014), Colombia aún presenta rezagos en esta área. $\mathrm{Al}$ año 2012, el $67 \%$ de los colombianos tenía por lo menos un producto bancario. A pesar de que el número de adultos pasó de 19,7 millones en diciembre de 2011, a 20,8 millones en diciembre de 2012, las cifras analizadas con detalle, demuestran que todavía hay problemas en la bancarización.

Pasamos del año 2007 del $55 \%$, es decir, de tener 28.100.679 a tener en el año 2012, un 67 $\%$, que indica 20.827 .472 cifra de crecimiento. $\mathrm{Si}$ nos detenemos en las cuentas de ahorro, veremos que las cifras no son tan alentadoras como inicialmente se puede entender. En la franja de cuentas de ahorro, entre el año 2007 y el año 2012, se pasó de 14.896.214 a 19.436.986, hay un incremento del $30 \%$.

Pero al analizar las cuentas de ahorro inactivas, nos encontramos con el dato de que, de estos 19,4 millones, 6,5 millones tienen la cuenta de ahorro cesante. En realidad solo 12,9 millones son quienes la usan con regularidad. Las razones que explican estos datos, estriban en la complejidad para acceder al banco y a los cajeros automáticos, así mismo en la dificultad para ingresar al sistema financiero por los trámites y los altos costos del mismo.

Es allí donde halla el nicho de mercado el servicio denominado DaviPlata. En este panorama de altos costos de infraestructura y de pago de personal, por las reglamentaciones gubernamentales, se hace necesario revisar el modelo tradicional de la banca que se venía prestando. Paradójicamente, Colombia tiene una de las más altas cifras de uso de teléfonos celulares, la penetración de este servicio es del $105 \%$ y la infraestructura de redes en la geografía nacional es del $100 \%$. De ahí, que aprovechar las ventajas de la telefonía celular terminó siendo una innovación tecnológica de valor agregado.

Es bueno precisar que el éxito de DaviPlata también es resultado de una nueva forma de entender las dimensiones del mercado y el concepto de banca tradicional. Lo normal del marketing es que se haga un grupo focal para evaluar un producto o servicio. Davivienda rompe estos esquemas, planteando un análisis de mercado más humano.

El equipo comando se desplaza a los barrios donde se asientan las poblaciones de la base de la pirámide. Iniciando una inmersión en el mundo de la cotidianidad, y generando vínculos personales, es así como se estudia lo que piensan los comerciantes minoristas, las cadenas de distribución, las expectativas del comerciante de la esquina, los obstáculos y metas de las cadenas de suministros y la logística de los camiones de distribución.

Mediante el contacto interpersonal, el diálogo vivencial y el entendimiento de la vida cotidiana y de los negocios, se construyen interpretaciones sobre las necesidades específicas de este segmento y sus limitaciones para la inclusión financiera. De igual modo, estas experiencias locales, se contrastaron con los procesos globales de inclusión financiera en África, Asia y América Latina. Es gracias a esta manera peculiar de trabajo etnográfico como nace DaviPlata. Una propuesta que rompe los paradigmas tradicionales de la banca.

Ruptura paradigmática número uno: simplicidad en el uso. Los productos bancarios 
son en exceso complicados y no producen en los clientes recordación emocional positiva. De ahí, que la sencillez y simplicidad de DaviPlata sea su valor agregado fundamental.

DaviPlata asume como reto desarrollar un servicio que podría ser utilizado por cualquier tipo de cliente (en bancos, sin servicios bancarios o con manuales) y al mismo tiempo, ser muy fácil de usar, por ende, se ofrece a la base de la pirámide, productos y servicios que pueden estar al alcance de sus necesidades. El solo sitio web de DaviPlata, demuestra la cercanía con la base de la pirámide: el portal transaccional se dirige comunicacionalmente a este segmento de la población.

Ruptura paradigmática número dos: modelos de precios. Como ya se indicó, los altos costos de los servicios bancarios explican la distancia con los contextos propios de las comunidades en la base de la pirámide y precisan una de las mayores quejas de esta población, en particular para los segmentos poblacionales que ganan salarios más bajos, así como para los asalariados de ingresos medios y altos, los servicios financieros se constituyen en un costo transaccional difícil de asumir. Frente a esto, proponer un servicio casi gratuito es una ruptura paradigmática total.

La propuesta de DaviPlata implica un rehacer las prácticas bancarias, en función de su público objetivo. Son varios procesos que se redefinen con la metodología de trabajo de la innovación que adoptó el Grupo Bolívar en el año 2007, llamada Systematic inventive thinking (SIT).

Esta metodología tiene sus fuentes en la teoría para resolver problemas de inventiva (TRIZ, por su sigla en ruso), la cual se emplea ampliamente para el desarrollo de nuevos productos, servicios y procesos, así como para la resolución de problemas, el análisis de las estrategias deresolución de conflictos y el estudio de las diferentes estrategias comunicacionales.
Con este modelo de innovación, se desarrolla el método "restar", se toma un proceso a innovar y se formula la pregunta: $¿$ de los elementos que componen estos procesos qué se puede restar? Al analizar con detenimiento el proceso para la apertura de una cuenta el resultado es el siguiente:

- Sucursales.

- Tarjeta débito.

- Formularios.

- Firmas y huellas dactilares.

- Papel.

- Comisiones o cualquier tipo de carga.

Prácticamente suprimir estos factores para generar inclusión se consideraba imposible. Pero DaviPlata demuestra que se pueden hacer rupturas revolucionarias en la forma de entender los negocios. En abril de 2011, DaviPlata se estrena como un servicio que podía ser usado por una persona desde su teléfono celular, activado desde su tarjeta SIM. En sus inicios, lo transaccionalmente permitido fue:

- Transferencias de persona a persona, DaviPlata a DaviPlata o DaviPlata de ahorro o cuentas corrientes.

- En efectivo, solo de Davivienda. Consignar en cuentas corrientes y pagos a granel.

- Pagos de cuentas, pagos de servicios públicos y pagos privados.

- Retiro de dineros por cajero ATM.

Todo ello sin necesidad de utilizar los canales tradicionales de apertura de cuentas. El proceso se realiza por teléfono, esto reduce los costos de una manera ostensible. La apertura de la cuenta y la verificación de datos del cuentahabiente, se hacen mediante el cruce de datos en las bases de información del gobierno nacional $\mathrm{y}$ en las bases internacionales, lo que previene el lavado de dinero y el uso ilícito del servicio.

Posteriormente, DaviPlata sigue al comportamiento del uso de las cuentas, lo que permi- 
te entender el manejo concreto de las mismas y las tendencias de los usuarios, si se detectan anomalías, se interviene en el caso específico. Esto se simplifica debido a que DaviPlata se enfoca en comunidades beneficiadas de subvenciones, personal activo y pensionado de las fuerzas militares, microcomerciantes y personas que reciben remesas. Los resultados saltan a la vista.

- Cero costos para los clientes.

- Retiro de dinero en ATM sin tarjeta débito.

- Giros para todos, en línea y sin costo para los clientes.

- Eficiencia en costos para el pago de subsidios del gobierno.

Hoy por hoy DaviPlata es el primer producto financiero en Colombia para el manejo del efectivo electrónico desde el celular, que posibilita realizar los siguientes servicios:

1. Activación desde el celular: no presencial, sin papeles y el registro es inmediato.

2. Consignaciones de efectivo: cuenta Davivienda, permite efectuar consignaciones en oficina, desde otro DaviPlata, puede realizar dispersiones masivas, y puede recibir o enviar remesas internacionales.

3. Hacer pagos: permite hacer pagos de servicios públicos y privados, compras en comercios y recargas de celular.

4. Retiros en efectivo: permite retiros sin tarjeta débito con acceso inmediato.

Sumado a lo anterior se cuenta con aliados estratégicos:

- Empresas de telecomunicaciones: Claro, Tigo y UFF.

- Punto Red y Edatel Telecomunicaciones para el caso de las redes.

- Soporte brindado por Redeban Multicolor, Gemalto, Cifin, BlueMessaging y Sistemcobro.
Los indicadores de resultados son elevados para el tiempo que lleva operando el servicio. Más de 1.800.000 colombianos usan DaviPlata. Posee cifras récord de bancarización. En solo tres meses abrió dos mil puntos DaviPlata. En dos meses vinculó 920.000 clientes.

En solo un día produjo 70.000 vinculaciones. Generó 646.000 pagos en tiendas de subsidios en diez días. Presentó cuatro millones de transacciones en el último mes de operación (Davivienda, 2013). De los cuales, el $77 \%$ no eran clientes, y el $61 \%$ de ellos no estaban bancarizados (Kotler et al., 2013, pp. 110-111). Además de esto presenta los siguientes resultados en sus target de mercado:

Subsidios: un millón a través de DaviPlata en 394 municipios de Colombia: Fondo de Prevención y Atención de Emergencias (Fopae), Servicio Nacional de Aprendizaje (Sena), Instituto Colombiano de Bienestar Familiar (ICBF), Familias en Acción y Jóvenes en Acción. Así mismo, tiene presencia en comunidades indígenas del Amazonas, Chocó, Risaralda y Tolima.

Fuerzas militares: ochenta mil pagos a través de DaviPlata a la Armada Nacional, la Fuerza Aérea, la Policía Nacional y el Ejército Nacional. Un total de 35.000 personas de las fuerzas militares usan el servicio DaviPlata.

Pagos masivos: más de 1.082 empresas vinculadas (Kotler et al., 2013, pp. 110-111). Entre estas: SsangYong, Empresas Públicas de Medellín (EPM), Avon, Universidad Nacional, Estibol, Corferias, Palmicultores de Tumaco, Sociedad de Vigilancia Privada de Agentes en Uso de Buen Retiro (Sovip Ltda.), Cooperativa de Caficultores del Occidente de Antioquia y Soem Temporal SAS. Un total de 350.000 pagos por medio de DaviPlata.

Giros nacionales: en el mes de agosto del año 2012 se inició el servicio con cinco mil giros nacionales mensuales, al día de hoy se están realizando 55.000 mil giros nacionales 
mensuales. La red de aceptación de estos giros, es de más de 1.500 cajeros, 550 oficinas y más de dos mil puntos de DaviPlata. Gracias a DaviPlata 800 mil colombianos presentaron inclusión financiera al usar, por primera vez, un producto financiero. Precisan sobre los niveles de inclusión al respecto Kotler et al. (2013):

El desarrollo de DaviPlata ha brindado al Banco Davivienda la oportunidad de acceder a segmentos de la población que no estaban vinculados al sector financiero, conocer de cerca sus necesidades y entender cómo las microfinanzas [forman] parte del engranaje económico del país. Gracias a este proyecto, Davivienda tiene ahora presencia en 700 municipios de Colombia. De ellos, en 379 no contaban con oficinas y 74 no tenían representación de ningún banco (pp. 110-111).

DaviPlata demuestra que cumpliendo una función social, se puede hacer dinero. La experiencia de DaviPlata concuerda con el anhelo de bien común esbozado por Felber en la entrevista al diario El País de España (2013), cuando señalaba el papel social de los bancos: "a largo plazo, la idea es que todos los bancos estén orientados al bien común, igual que los colegios, los institutos [y] las universidades".

Las lecciones que podemos aprender de DaviPlata, son aprendizajes que se recomiendan a aquellas corporaciones que creen que no es posible hacer capital con una visión práctica de lo social. Por ello, la primera enseñanza es que sí puede existir una armonía práctica entre lo social y lo económico. Kotler et al. (2013) expresan que es factible concertar lo social y lo económico, sencillamente para estos autores, hacer lo bueno, funciona:

El compromiso con hacer lo bueno o lo mejor no es una garantía de éxito en el mercado, pero en los últimos 30 años numerosas empresas han demostrado que pueden construir un mundo mejor y ganar dinero al mismo tiempo. La experiencia también demuestra que para diseñar iniciativas sociales de marketing y corporativas de éxito hace falta inteligencia, compromiso y delicadeza (p. 21).

La segunda lección se orienta hacia el enfoque que ofrece el marco teórico del concepto de creación de valor compartido explicado por Porter y Kramer (2011). Para estos estudiosos la visión estrecha del capitalismo ha minado la legitimidad de las empresas en la opinión pública y ha limitado su crecimiento.

Al basar únicamente su desarrollo en la amplitud de mercados y en el lucro, sin tener en cuenta las externalidades producidas por sus acciones y obviando las necesidades de su entorno, el capitalismo ha propiciado las condiciones que a largo plazo no lo harán sostenible.

Se entiende por creación de valor compartido, las diferentes estrategias organizacionales que optimizan en la praxis la competitividad de la empresa, de forma armónica enlazadas con las mejoras de las condiciones reales económicas y sociales en las comunidades donde tiene presencia la compañía.

Si analizamos el impacto de DaviPlata podemos apreciar objetivamente, que hay un equilibrio y una transformación desde las microfinanzas y desde el mejoramiento de los entornos. En este orden de ideas, la creación de valor compartido es la segunda gran lección. Se puede crear una firma sostenible si se tienen en cuenta las necesidades del entorno donde la organización se desenvuelve.

DaviPlata se apalanca sobre la base de las necesidades que arrojan sus diagnósticos sobre el contexto, para desarrollar inclusión social, con las personas, desde las personas y para las personas.

La tercera lección es la importancia de la innovación social. La creencia de que se deben innovar servicios o productos y crear océanos azules donde la competencia no pueda llegar, está arraigada en la mentalidad actual. Desde 
que Chan Kim y Mauborgne (2005) plantearon la necesidad de crear océanos azules donde la competencia no pudiera llegar, y apartarse de los océanos rojos, donde los productos y servicios genéricos terminaban arrasando los mercados, las entidades se concentraron en crear innovaciones tecnológicas de valor agregado. Lo que podemos aprender de DaviPlata es que la innovación que considera la inclusión social, también es la creación de un océano azul.

No solamente son servicios y productos pensados para mercados lucrativos, sino también productos y mercados concebidos para la base de la pirámide en función de la innovación tecnológica que agrega valor y que construye valor compartido. Chan Kim y Mauborgne (2005) señalan al respecto:

La innovación en valor es la piedra angular de la estrategia del océano azul. Le hemos dado ese nombre porque en lugar de girar alrededor de la victoria sobre la competencia, el objetivo es lograr que esta pierda toda importancia al dar un gran salto cualitativo en valor tanto para los compradores como para la compañía, abriendo de paso un espacio nuevo y desconocido en el mercado (p. 17).

Las innovaciones de valor también se pueden hacer en función de lo social. Los negocios inclusivos manifiestan lo factible de pensar en lo social, de repensar lo social y de ser alternativa de cambio frente a lo social.

\section{Merquemos Juntos: resiliencia solida- ria de las víctimas de la violencia}

La resiliencia es un concepto novedoso en el ámbito de la psicología, que tiende a proyectarse con fuerza en los campos de análisis de otras ciencias sociales como la sociología, la antropología, la política, la ecología y en particular, ingresa con mucho vigor al ámbito de lo empresarial (Schneider, 2007).

La resiliencia es un campo interdisciplinar de estudio, que con una mirada holística da cuenta de cómo se puede rearmar lo desarmado en los ámbitos micro y macro. La palabra denotaba metafóricamente la capacidad interna que poseen las estructuras físicas de soportar un peso para el que no fueron diseñadas, y volver a la posición original.

Este fenómeno del mundo de la ingeniería, demarcó el estudio de la resiliencia como la capacidad interior de las personas, los grupos sociales y organizaciones, de enfrentar la adversidad y recuperarse con las fortalezas internas. La muestra de la forma como se usa el concepto de resiliencia la encontramos en la definición integral y metadisciplinaria que nos brinda el analista de sistemas empresariales Andrew Zolli y Healy (2012):

En el mundo de los negocios, el término suele usarse en referencia a la implementación de copias de seguridad (de datos y recursos) para garantizar la continuidad de las operaciones ante desastres naturales o desastres producidos por el hombre. Aunque el énfasis en cada caso es diferente, cada una de estas definiciones se basa en uno de los dos aspectos esenciales de la resiliencia: la continuidad y la recuperación ante el cambio (p. 16).

La resiliencia por ende implica un conjunto de destrezas cognitivas que le posibilitan a una persona, grupo, comunidad o empresa, enfrentar las diferentes adversidades, cambios y contingencias que surgen redefiniendo el contexto en el que se encuentran inmersos estos sujetos sociales.

Una expresión cercana a la resiliencia es la de reconstrucción emocional. Los estudiosos de la resiliencia (Cyrulnick, 2011; Kotliarenco \& Cáceres, 2011; Seligman, 2005), insisten en determinar o precisar qué factores son los que propician la resiliencia. Entre estos: el sentido del humor, la religiosidad, la vida familiar, la idea de futuro, la capacidad para proponer futuros alternos, la creatividad y el optimismo.

Desde este escenario de análisis, y centrándonos en el aspecto económico y empresa- 
rial, uno de los primeros en contrastar el estado de la economía en América Latina y las diferentes prácticas de supervivencia de las empresas latinoamericanas en entornos de incertidumbre y cambio constante, con el concepto de resiliencia, fue el analista peruano Ben Schneider (2007).

En su detallado estudio sobre la resiliencia y en especial de los factores que posibilitan construir empresas en contextos de inestabilidad, Schneider (2007) cita la importancia de las creencias de la organización, de la táctica y de la estructura que se logre crear desde la alineación estratégica; y examina casos de macroempresas que lograron enfrentar la incertidumbre y las diferentes crisis económicas, en particular ilustra el fenómeno al explicar los casos de las firmas: Tequila Cuervo La Rojeña en México, Los Grobo Agropecuaria en Argentina, Farmacias Ahumada en Chile, Grupo Carvajal en Colombia, Grupo Wong en Perú y Lorenzo Zambrano en México.

De ahí que miremos la resiliencia como una decisión personal, grupal e institucional y una actitud frente a la vida, es una forma de pensar, sentir y actuar conexa con el concepto de proactividad. Desde la perspectiva de Covey (1997), la proactividad es nuestra capacidad de elegir la forma de sentirnos, nuestra singular y única capacidad de respuesta frente a los estímulos externos.

En ese sentido, la resiliencia es una proactividad, dado que las personas deciden cómo sentirse respecto a los estímulos externos que no les son favorables. En esta dimensión es pertinente la afirmación de Cyrulnick (2011) sobre el poder efectivo de la resiliencia:

[...] la capacidad que tiene un ser humano (niño, adolescente, adulto), a pesar de sus dificultades personales (físicas, mentales y psíquicas), para intuir y crear, en circunstancias desfavorables o en un entorno destructor, respuestas que le hacen posible no dejarse atrapar por las circunstancias y dificultades personales ni por las condiciones del entorno sino por el contrario, proponer y realizar comportamientos que le permiten una vida con menos sufrimiento y encontrar un lugar en su medioambiente, lo cual le permite desarrollar sus capacidades y anhelos (p. 36).

Una de las claves para proyectar resiliencia en la comunidad es la posibilidad de empoderar a los grupos y comunidades. Se resalta el concepto de empowerment, que es la habilidad de permitir autonomía y autogestión personal y comunitaria (Blanchard, 2006). Es a través del aprendizaje en la autonomía, como se puede romper el problema de la beneficencia y de la espera, es dotando a los grupos del poder de decidir, como se manifiesta de manera real y duradera los rasgos resilientes. En el escenario comunitario y social, las formas solidarias son portadoras de resiliencia.

Un caso ejemplar de este proceso es el que protagoniza Merquemos Juntos. Esta organización es una asociación de desarrollo comunitario, que en medio de un contexto social altamente conflictivo, se atreve a proponer y a construir nuevos modos para afrontar el conflicto y asumir con resiliencia, proactividad y solidaridad, la difícil tarea de jalonar proyectos que impliquen una transformación autónoma de sus asociados.

A través del microcrédito, Merquemos Juntos se convierte en una sólida opción de propuesta urbana. La historia de su surgimiento señala la resiliencia que emana de la solidaridad. Esta historia, narrada a manera de etapas, se toma directamente del portal de Internet de la asociación, y es altamente valiosa, ya que se cuenta desde la visión de las asociadas y asociados de esta organización, que en la actualidad se constituye en un caso exitoso de negocio inclusivo (Merquemos Juntos, 2013).

Hoy este programa, tiene tanta aceptación en la comunidad que hasta el momento se ha convertido en un activador de la economía familiar popular, que da cursos de preparación a las pequeñas empresas existentes, las organiza 
en redes y las vuelve sostenibles. Esto se consigue porque Merquemos Juntos es un centro generador de confianza y de "cómo hacer las cosas" a partir de recursos mínimos.

En el año 2012, los éxitos de Merquemos Juntos le hacen ganar el Premio Nacional de la Paz, pero este es uno de los tantos logros que ha cosechado esta experiencia de solidaridad en medio del conflicto y de la escasez de los recursos mínimos y esenciales para vivir dignamente en comunidades marginales. En 1992 Merquemos Juntos se propone solucionar un problema de alimentos y en menos de ocho años y mediante la búsqueda de opciones y de inversión internacional, ha creado intraempresas: pastelería, panadería, tienda de abarrotes, taller de confecciones y planta de procesamiento de alimentos.

Estas unidades de negocios las operan los mismos asociados, quienes a través de procesos de capacitación, han logrado desarrollar principios propios de la autogestión. En el presente Merquemos Juntos compra mensualmente más de dos toneladas de alimentos y es una opción alimenticia para las comunidades que viven en la pobreza absoluta. Cabe aclarar, que estos negocios crecen paulatinamente en la medida en que se posicionan sus diversas intraempresas. Sus líneas de actuación, que la sitúan como negocio inclusivo son:

1. Restaurante: uno de los objetivos iniciales de la asociación era solucionar el problema de la alimentación diaria, por ello el programa de los restaurantes, brinda un plato de sopa diario a las personas más necesitadas de la comunidad. Este alimento tiene un precio simbólico de \$500. El almuerzo corriente se vende a un costo de $\$ 3.500$. Este proyecto se refuerza con el programa de complemento alimentario.

2. Complemento alimentario: proyecto que se adelanta en asocio con la Al- caldía de Barrancabermeja, Colanta, y busca dar un acompañamiento nutricional a los niños y niñas de bajos recursos. En este momento la cifra de refrigerios suministrados diariamente es de 26.000, que día a día se entregan a los menores pertenecientes a una población vulnerable nutricional y socioeconómicamente, de las áreas rural y urbana del municipio de $\mathrm{Ba}-$ rrancabermeja. El refrigerio escolar se compone de un complemento alimentario y comprende un panificado y una bebida láctea (Merquemos Juntos, 2013).

3. Panadería: Merquemos Juntos inicia la construcción de la panadería con recursos que nacen de la solidaridad de sus asociados, y capacita y emplea a personas miembros de la asociación. Esta panadería vende el pan a la módica suma de $\$ 200$ y la bolsa de pan en toda su variedad no sobrepasa los $\$$ 1.000. Actualmente la panadería produce 20.100 panes diarios.

4. Confecciones: en el edificio de la sede se construyó un taller de corte y confección de ropa. En este momento la unidad de negocio se dedica a la producción industrial de uniformes escolares, uniformes de trabajo y pedidos acordes con las diferentes temporadas del año y necesidades de los clientes. Es una opción de empleabilidad que maneja la asociación.

5. Tienda escolar: frente a la necesidad de conseguir buenos precios para los asociados en lo concerniente al material escolar, se abre la tienda escolar que persigue brindar todo lo necesario para el estudio a precios muy bajos.

6. Crédito estudiantil: apoyo en el pago de matrículas a estudiantes con el 
otorgamiento de cupos crediticios, dándole prioridad a los estratos 1 y 2 , con interés mínimo y sin cobro de comisión.

Pero lo que hace exitosa su visión solidaria y la posiciona en los primeros puestos de negocios inclusivos, es el tema de los microcréditos, en el cual están alcanzando altos niveles de inclusión social y de desarrollo de organizaciones empresariales.

Los indicadores de microcrédito demuestran un incremento del negocio y una efectividad en la recuperación de cartera. Según la Agencia de los Estados Unidos para el Desarrollo Internacional (Usaid, por su sigla en inglés) (2010), el desempeño microfinanciero de Merquemos Juntos ha beneficiado a más de cuatro mil familias. Realizó en solo un año de actividades, 1.006 préstamos (información del 2010), con un riesgo de cartera del $15 \%$. De estos préstamos el $65 \%$ se concede a mujeres; $5 \%$ a jóvenes y $20 \%$ a clientes rurales. Lo interesante es que del volumen de personas que prestan dinero, el $70 \%$ de las mismas ingresa a un programa de ahorro voluntario.

El éxito del microcrédito, radica en la forma en que se implementa el servicio y los niveles en que se desarrolla el mismo. Los montos de los créditos otorgados están entre los $\$ 300.000$ y el $\$ 1.500 .000$. Si llevamos esto a la expresión de los salarios mínimos, nos encontramos con que los microcréditos oscilan entre 0,7 smmlv y 3,7 smmlv.

Los préstamos se dan con un plazo de entre cuatro y veinticuatro meses dependiendo del valor asignado. Hoy por hoy la asociación maneja más de dos mil millones de pesos en capital, que favorecen alrededor de 1.800 personas entre: población estudiantil, vendedores informales y comerciantes asentados en las plazas de mercado, tiendas, misceláneas, almacenes pequeños y asesores de ventas por catálogo.
La tasa de crédito es de $2,5 \%$, tasa nominal mensual sobre el saldo del capital. El procedimiento del crédito inicia con una visita de verificación de existencia de la empresa. Otra característica clave de sus microcréditos, es la flexibilidad de los requisitos para el préstamo de los mismos. Declara el contador de Merquemos Juntos, al respecto de las garantías y exigencias para realizar un microcrédito (PNUD, 2010):

[...] la primera, un trabajo sano, un salario, es suficiente. La segunda, un certificado de tradición y libertad de la casa que puede estar del puente para acá [se refiere a la ubicación de la vivienda en una de las comunas, nororiental o suroriental de Barrancabermeja] -que no se recibe en bancos, solo del puente para allá-. La tercera, el certificado de Cámara de Comercio: el negocio, su tamaño y su acreditación (p. 202).

Podemos mencionar varios aprendizajes que se desprenden de la forma en que la asociación de desarrollo comunitario, Merquemos Juntos, maneja el tema de los microcréditos. El aprendizaje número uno es la superación de la disyuntiva entre flexibilización de requisitos para el crédito y elevación del riesgo de recuperación de la cartera.

El pensamiento tradicional indica que las personas pobres no devuelven el dinero que se les presta. Y que, por lo tanto, es necesario asegurar la forma de recuperar el capital. Así, surge un círculo difícil de romper para quienes viven en situación de marginalidad: para poder conseguir dinero de instituciones financieras es vital probar que se tiene dinero. En este aspecto Merquemos Juntos, se aparta de la mirada convencional y se apoya en el desarrollo de un valor capital para ellos como empresa solidaria: la confianza.

Así mismo, un valor agregado de Merquemos Juntos es la flexibilización del pago de la cuota, supeditando este al flujo de caja que presente la empresa deudora. Ello implica que se puede sufragar la cuota en diversas modalidades, desde el día a día, hasta el desembolso convencional. Si el deudor se atrasa, se le acepta lo que 
pueda pagar y dado el caso, se pasa a un congelamiento de los intereses. Este apunte lo encontramos en la narración de uno de los asociados de Merquemos Juntos (PNUD, 2010):

[...] ante un banco convencional, por experiencia propia, lo primero que exigen es que usted tenga una cuenta abierta con por lo menos 50.000 pesos, con tarjeta, y cuotas para todo: estudio del crédito, estudio de títulos, desembolso... Merquemos Juntos, por el contrario, se preocupa por el trato a las personas: el monto del crédito es como se ha solicitado: si son 100.000 son 100.000 , no se incrementa con comisiones y pagos adicionales. Se le entrega el dinero al beneficiario, no a terceros (p. 204).

La segunda lección es la necesaria construcción de relaciones de género. El éxito de los microcréditos gira en torno al papel destacado de la mujer en la gestión empresarial. Es claro que el préstamo recaiga en mujeres, pues muchas de ellas son víctimas de la violencia y por lo tanto, son madres solteras o viudas que deben agenciar su propio negocio.

Lo que resalta es la capacidad de autogestión y administración de los recursos, empresarialmente hablando, de la parte femenina. Las mujeres destinan un porcentaje de sus ganancias al mejoramiento de las condiciones de vida de sus hijos y de su negocio. Es decir, el dinero se reinvierte en el bienestar de la familia. Al respecto, una beneficiaria de este tipo de crédito relata su experiencia (PNUD, 2010):

Mi meta es el próximo año la casa entera. En qué forma uso yo mi ahorro: hace años no tenía casa, ni familia. Construyo mi casa, la levanto. Merquemos Juntos me da la posibilidad de enlazarnos con el trabajo de nosotros, de mi esposo y mío, tengo mi niño saludable, mi familia satisfecha, mi casa empezó por muy poco: ahora tengo fotocopiadora, mi proyecto es un computador; esta es la demostración (p. 204).

Un tercer aprendizaje radica en el acompañamiento a los procesos desde una visión educativa. Una de las grandes falencias de las personas que emprenden un micronegocio es el total desconocimiento del mismo. Si se direccionan microcréditos a comunidades marginales, se hace imperativo dotarlas de las herramientas teórico-conceptuales que les posibilite afianzarlos y amplificarlos para que no se sucumba al inicio de la idea de negocio.

Ello implica diseñar programas de capacitación que potencien las habilidades y competencias básicas para ser empresarios. En resumidas cuentas, Merquemos Juntos presta el dinero, pero, paralelo a esto, diseña programas de capacitación permanente para que fructifique la empresa o famiempresa. El revisor fiscal de Merquemos Juntos expresa (PNUD, 2010):

Cuando se está capacitando, la tendencia es subvalorar el conocimiento del capacitado: nosotros partimos de ese conocimiento para mejorarlo. Tienen que sentirse empresarios; nosotros solo somos facilitadores para que ellos organicen su negocio... no más. En capacitación solo les damos un 'brochazo': esto es lo que usted necesita para mejorar su negocio pero después vamos al negocio y sobre el terreno verificamos y afinamos la capacitación, ya en la práctica, tal como el uso de los libros de contabilidad, ahí se les explica para qué sirve (p. 205).

Visto así, las capacitaciones se constituyen en oportunidades de mejora y cambio continuo, y señalan que no basta con propiciar los primeros recursos para un apalancamiento o consolidación del proyecto empresarial. En este sentido, se precisa que la resiliencia es posible si se atiende holísticamente la dimensión del ser. La educación por ende, se convierte en uno de los vértices de la construcción de una visión integral para superar efectivamente los círculos viciosos de la pobreza.

\section{Conclusiones}

Las dos experiencias sistematizadas en este trabajo, son limitadas frente a la magnitud del movimiento de negocios inclusivos en Colombia, que cada vez toman mayor fuerza. 
Una muestra de ello, es el texto de Kotler, et al., (2013). Llama poderosamente la atención que en este libro se haga una especie de síntesis especial sobre las diferentes estrategias de RSE en Colombia. Consideramos que esto no es casual, es más bien producto del crecimiento de esta tendencia dentro de los indicadores nacionales. Los trece ejemplos que plantea Kotler et al., (2013) son los siguientes:

- Los negocios inclusivos desarrollados por el Grupo Empresarial Bolívar en el ámbito de las copropiedades.

- DaviPlata y su servicio de bancarización.

- Programa Medioambiental del Politécnico Grancolombiano.

- Alpina y su proceso de empoderamiento comunitario para mejorar procesos de producción.

- El programa de desarrollo de proveedores locales de Pacific Rubiales Energy.

- Nestlé y su proceso de creación de valor compartido con proveedores en el área rural.

- Fundación Bavaria y su aporte al desarrollo empresarial.

- El programa de trabajo voluntario de Telefónica Movistar y su programa de Internet móvil para comunidades marginales.

- Nutresa y su proceso direccionado a mejorar la calidad de la educación.

- La multinacional Phoenix y su programa de reciclaje.

- La Industria Licorera de Caldas y su trabajo por el medioambiente y la comunidad.

- Ecopetrol y su compromiso medioambiental por el aire limpio.

- Unilever y el plan de vida sostenible, huertas comunitarias y materias primas de fuentes sostenibles.

A pesar de lo anterior, Kotler et al. (2013), se mueven en un marco conceptual bastante amplio. Para ellos, filantropía, voluntariado, empoderamiento comunitario y negocios inclu- sivos son simples manifestaciones de RSE. Lo que se puede constatar a partir del seguimiento de estos casos, es que el concepto como tal, necesita ensancharse y se debe dimensionar cada una de sus aristas para precisar a qué tendencia de la RSE corresponde.

Es por ello que, una de las conclusiones de este trabajo se orientó a precisar las características de los negocios inclusivos y las tendencias implícitas en estos, según Márquez, Reficco y Berger (2009) los negocios inclusivos debían presentar:

- Transformación del statu quo.

- Motivación comercial y rentabilidad económica.

- Conexión entre lo global y lo local.

- Innovación paciente.

- Apalancamiento en recursos locales.

- Protagonistas nuevos y más diversos.

A estas características agregamos dos nuevas: resiliencia y economía del bien común. Es interesante que cada una de estas ocho particularidades pueda estar contenida en los negocios inclusivos señalados en este artículo, especialmente en los dos casos sistematizados como exitosos.

Lo cierto es que, al año 2013, la economía colombiana presentó unas particularidades de desarrollo y de panorama optimista, pero la deuda social sigue pendiente. El escenario de un futuro posible de posconflicto, exige una renegociación de lo público y lo privado, donde lo privado sea capaz de redireccionarse al ámbito de lo social en una perspectiva de ganarganar o no hay negocio.

Las promesas pendientes de la globalización podrían empezar a cumplirse, si el capitalismo es capaz de mirar las oportunidades en la base de la pirámide y asumir la concepción de que en este mundo hay suficiente para todos. Pero ello implicaría abandonar el egoísmo y la ambición propios del capitalismo. Por ende, 
uno de los pilares sobre los cuales se deben estructurar los negocios inclusivos en el futuro es el del rescate del valor de la solidaridad y de la mentalidad de la abundancia. Se finaliza esta investigación con una cita que hace Kotler et al. (2013), de Pringle y Thompson:

Los consumidores están llegando a la cúspide de la pirámide de necesidades de Maslow y buscan la autorrealización. Lo que buscan y les atrae ahora son las demostraciones del bien. En un sentido antropomórfico, si los consumidores ya saben cómo funciona una marca y cómo piensa y siente, la siguiente pregunta es ¿en qué cree? (p. 41)

\section{Referencias}

Banco de comercio exterior [Bancóldex]. (2013). Cronica sobre DaviPlata. Recuperado de http://www.sevenoticias.com/ sn/jo1515/index.php?option=com_conte nt\&view=article\&id=545:DaviPlata-manejo-de-efectivo-a-travesdel celular\&cati $\mathrm{d}=36$ : noticias\&Itemid $=54$

Blanchard, K. (2006). Empowerment: tres claves para que el proceso de facultar a los empleados funcione en su empresa. Bogotá: grupo editorial Norma.

Chan-Kin, W., \& Mauborgne, R. (2005). La estrategia del océano azul. Bogotá: Grupo Editorial Norma.

Covey, S. (1997). Los 7 hábitos de las personas altamente efectivas. Paidos: Barcelona.

Cyrulnik, B. (2011). Morirse de vergüenza. Barcelona: Debate.

Davivienda. (2013). DaviPlata. Recuperado de https://productos.davivienda.com/PersonasyFamilias/DaviPlata.aspx

Departamento de Prosperidad Social [DPS]. (2012). Crónica "Merquemos Juntos", al- ternativa de esperanza para familias desplazadas del Magdalena Medio. Recuperado de http://www.dps.gov.co/contenido/ contenido. $\operatorname{asp} x ?$ conID $=6706 \&$ cat $I D=127$

Felber, C. (2012). La economía del bien común. Barcelona: Deusto.

Felber, C. (01 de septiembre de 2013). Un mundo sin capitalismo. El país, España. Recuperado de http://internacional.elpais. com/internacional/2013/08/30/actualidad/1377859146_576653.html

Frankl, V. (1991). Hombres en busca de sentido. Barcelona: Herder.

Henao, M., Borja, W., Mantilla, J., Garcia, L., \& Ordoñez., F. (2014). Reporte trimestral de inclusión financiera (Informe técnico). Recuperado del sitio de internet de Asociación Bancaria y de Entidades Financieras (Asobancaria): http:// www.asobancaria.com/portal/pls/portal/ docs/1/4394197.PDF.

Kotler, P., Hessekiel, D., \& Lee, N. (2013). Lo bueno Funciona: "explica cómo las iniciativas de marketing social construyen un mundo mejor y favorecen la cuenta de resultados". Bogotá: ediciones de la $\mathrm{U}$.

Kotliarenco, M. A., \& Cáceres, I. (2011). Estado del arte en Resiliencia III. Diplomado en promoción de la parentalidad positiva. Estrategias de evaluación e intervención eco-sistémica. Recuperado de https:// www.uai.edu.ar/transferencia-universitaria/aprendizajeyservicio/ESTADO $\% 20$ DE\%20ARTE\%20EN\%20RESILIENCIA $\% 20 \% 20$ Organizacion $\% 20$ Panamericana $\% 20 \mathrm{de} \% 201 \mathrm{a} \% 20$ Salud $\% 20 \% 20$ Organizacion $\% 20$ Mundial $\% 20 \mathrm{de} \% 20$ la\%20Salud.pdf

Latorre, E. L. (enero, 2010). Comunicación, Memoria y Resiliencia. Estudio de la me- 
moria de las víctimas del conflicto armado en el Departamento del Magdalena: presentificación, visibilización, catarsis y resiliencia. Prolegómenos. Derechos y Valores 13(25), 95-109.

León, M., Baptista, M., \& Contreras, H (mayo, 2012). La innovación social en el contexto de la responsabilidad social empresarial. Fórum Empresarial, 17(1), 31-63.

Licandro, O. (julio, 2009). Negocios inclusivos: una estrategia eficiente de Responsabilidad Social Empresarial para colaborar en la lucha contra la pobreza. Revista Plaza financiera, 8(1), 90.

Licandro, O., \& Pardo, L. (2013). Experiencias de negocios inclusivos en el Uruguay. Montevideo: Universidad Católica del Uruguay; Fundación AVINA.

Márquez, P., Reficco, E., \& Berger, G. (junio, 2009). Negocios inclusivos en América Latina. Harvard Bussines Review, 87(5), 28-38.

Merquemos Juntos (2013). Merquemos Juntos. Asociación de desarrollo comunitario. (párr. 4). Recuperado de http://www. etpbarranca.org/merquemosjuntos/Archivos /Hicimos.html

Porter, M., \& Kramer, M. (enero, 2011). La creación de valor compartido. Revista Harvard Business Review América Latina. Recuperado de http://www.filantropiatransformadora.org/attachments/article/198/Shared $\% 20$ Value $\% 20$ in $\% 20$ Spanish.pdf

Prahalad, C. K. (2008). La oportunidad de negocios en la base de la pirámide: Un modelo de negocio rentable, que sirve a las comunidades más pobres. Bogotá: Grupo Editorial Norma.

Programa de las Naciones Unidas para el Desarrollo -[PNUD]. (2010). Rutas, caminos y experiencias de nuestra gente del
Magdalena Medio. Bogotá: Corporación de Desarrollo y Paz del Magdalena Medio.

Sandel, M. (2011). Justicia: ¿hacemos lo que debemos?. Barcelona: Debate.

Sandel, M. (2013). Lo que el dinero no puede comprar: los límites morales del mercado. Barcelona: Debate.

Schneider, B. (2007). Resiliencia: cómo construir empresas en contextos de inestabilidad. Bogotá: Grupo Editorial Norma.

Seligman, M. (2005). La auténtica felicidad. Barcelona: Ediciones B Senge, P. (1999). La Quinta Disciplina: cómo impulsar el aprendizaje en la organización inteligente. Barcelona: Granica.

Senn, A. (2008). El nuevo paradigma de los mercados financieros. Barcelona: Alianza Editorial.

Silva, V. (2009). Negocios Inclusivos o de base de pirámide: una oportunidad de desarrollo en el marco de la Responsabilidad social corporativa. Documento No. 1, Programa de ética empresarial y económica, Universidad Alberto Hurtado, Chile.

Singer, P. (1995). Ética para vivir mejor. Barcelona: Ariel.

Soros, G. (2008). El nuevo paradigma de los mercados financieros. Barcelona: Taurus.

Stiglitz, J. (2012). El precio de la desigualdad. Barcelona: Taurus.

Yunus, M. (2011). Las empresas sociales. Barcelona: Paidos.

Zolli, A., \& Healy, A. (2012). Resiliencia: por qué las cosas vuelven a su lugar. Bogotá: Carvajal Educación. 